\title{
How Does Collaborative Cheating Emerge? A Case Study of the Volkswagen Emissions Scandal
}

\author{
Christopher M. Castille \\ Nicholls State University \\ christopher.castille@,nicholls.edu
}

\author{
Andrew Fultz \\ Rutgers Business School \\ fultzae@gmail.com
}

\begin{abstract}
Since 2014, Volkswagen (VW) has been enthralled in a reputation-tarnishing cheating scandal that has raised questions regarding how collaborative cheating unfolds in organizational settings. While the behavioral ethics literature provides some insights, this literature is largely confined to individual decision makers and so little work examining how collaborative cheating emerges has been done. Therefore, with this case study, we draw on various data sources (e.g., court case summaries, investigative reporting, technical reports, popular press outlets, and publically available employee interviews) and use case study methodology (i.e., grounded theory, open-systems diagnostics) to construct a process model that explains how collaborative cheating emerges in organizational settings. Theoretical and practical implications are also discussed.
\end{abstract}

\section{Introduction}

Many researchers extol the virtues of collaboration in organizational settings [1,2]. Collaboration has been defined as a process by which individuals with various backgrounds come together and, through interaction, information sharing, and coordination of activities learn and, ultimately, perform [2]. Collaboration is often viewed as a vehicle that facilitates ongoing learning, results in innovative strategies for competing in the marketplace, promotes diversity, and other positive outcomes for organizations.

However, there are times in organizational life when individuals within a team setting decide to cheat, and coordinate their activities as needed (e.g., students sharing tests to help their peers, scientists using questionable research practices to ensure a study gets published). Though we understand what drives individuals to cheat, such as the facilitating conditions (e.g., social norms) and individual differences (e.g., moral disengagement) [4-9], what compels individuals to contribute to and sustain a collective cheating effort (i.e., collaborative cheating) is deserving of greater scrutiny than what has been done previously [e.g., 3]. This is partly because little research examines how unethical decision making occurs at the collective level [10]. One likely reason why little research exists on collaborative cheating is that studying it presents many problems that are not easily broached conventionally (e.g., experimental research), not the least of which involves gaining access to data on ethically and legally sensitive matters [11].

Given that organizations are increasingly relying on group decision making to compete in the marketplace, understanding what drives individuals to contribute to a collective cheating effort is critical. Recently, the Volkswagen emissions scandal, which involved many managers and engineers who helped create, implement, and conceal a device that would cheat emissions testing, provided scholars with the opportunity to understand how collaborative cheating emerges. Here, we use case study methodology to explain how collaborative cheating emerged at VW in order to form propositions regarding how this phenomenon emerges in organizational settings in general, providing direction for future study. To diagnose the causes of collaborative cheating at VW, we apply the open-systems model [12], a commonly used diagnostic framework in organizational research. This model acknowledges that organization's act on information in their larger environment, transforming inputs (e.g., human capital, materials) into outputs (e.g., goods, services, ideas) via transformation processes (e.g., social components, and technological components). How well these outputs satisfy constraints in the environment (e.g., regulatory needs and customer demands) serves as feedback for the organizational system, which can result in changes to the system or how it should be regulated. By applying this diagnostic model to our data, we hope to illuminate the numerous influences both inside and outside VW that contributed to the emergence of collaborative cheating.

We use case study methods for the following reasons. First, compared to quantitative approaches, 
qualitative methods flexibly capture the richness of collective constructs and processes in ways that can lead to generalizable and testable theory $[13,14]$. Second, the Volkswagen scandal has birthed a large volume of information on the inner-workings of $\mathrm{VW}$ leading up to the creation, implementation, and concealment of the defeat device, making these methods well-suited to the available data. Third, a case study allows us to form a complex yet detailed understanding of the role of context (e.g., regulations, culture, leadership) and causal mechanisms that shape the emergence of collaborative cheating behavior [15]. Researchers have suggested that we might learn something by studying specific cases of organizational failures [16]. Here, we seek to do just that by answering the following research question:

What are the mechanisms that explain how collaborative cheating, a form of collective and coordinated unethical behavior, emerges in organizations?

\subsection{An Overview of the Volkswagen Emissions Scandal}

Since 2014, VW has been embroiled in a cheating scandal that has tarnished its reputation. In 2014, researchers studying VW diesel-burning vehicles identified a large discrepancy between the emissions levels produced under laboratory and typical driving conditions [17]. This discrepancy was later attributed to a defeat device (software) created under the direction of VW management. This defeat device enabled equipped vehicles to respond to laboratory testing procedures by constraining emissions to levels deemed acceptable by the Environmental Protection Agency (EPA). When performing under typical driving conditions, equipped vehicles produced between five and thirty-five times the level of toxins deemed acceptable by the EPA [17]. Such excess pollution was predicted to cause approximately 59 premature deaths, 31 cases of chronic bronchitis, 34 hospital admissions, 120,000 days of restricted activity-including lost work days-210,000 days with lower-respiratory problems and 33,000 days with increased use of asthma inhalers. The overall cost burden would be approximately $\$ 450$ million [18].

In 2017, a court concluded that there was sufficient evidence indicating that certain VW managers $^{1}$ in power during the scandal (i.e., CEO of

\footnotetext{
${ }^{1}$ For anonymity, all names throughout the paper are replaced with generic identifiers (e.g., Executive A, Engineer A, etc.)
}

VW AG, CEO of VW Group of America, and CEO of the VW Passenger Cars brand) acted with scienter, which refers to a mental state embracing intent to deceive, manipulate, or defraud.

\subsection{Study Purpose}

We use qualitative research methods to examine key historical events in the VW scandal, namely the (1) creation, (2) implementation and refinement, and (3) concealment of the defeat device. We use this timeline to construct a process model describing how collaborative cheating emerges. Our data come from multiple sources (e.g., legal documents, investigative reporting, technical reports, popular press outlets, and employee interviews). The high-profile nature of the VW scandal has led (and continues to lead) to the creation of many documents, making it a prime candidate for analysis via case study $[15,19]$.

\section{Case Study Methods}

Following guidance from the literature [14,20], we began our case study with a focal concept collaborative cheating - to direct our analysis. We examine all relevant factors within the context of the VW scandal (i.e., management, culture, industry setting, organizational strategy, individual histories) in order to understand how individuals within VW conspired to cheat emissions testing. We define collaborative cheating as cheating that occurs when individuals of various backgrounds interact to create, implement, and sustain solutions to problems that violate ethical obligations or norms. This definition shares features of a commonly used definition of collaboration that we introduced earlier, such as the presence of a variety of expertise that is brought together via information sharing, shared creative problem solving, and coordination within the group [2]. However, collaborative cheating would involve factors that incline individuals to disregard ethical principles, or to morally disengage from their behavior $[6,21]$, in the pursuit of short-term self-interest. Collaborative cheating is emergent and involves sharing knowledge and expertise. However, agents contributing to a collective cheating effort recognize ethical wrongdoings and demonstrate a willingness to disregard such obligations and rationalize their behavior (e.g., for the greater good of their company, their own self-interest, or both). It also differs from individual cheating, which involves no voluntary assistance from others (e.g., cheating on a test by 
keeping correct answers written on a notecard that is taped to the bill of a baseball cap).

\subsection{Data Collection}

Data were collected in early 2017. We gathered literature on the VW emissions scandal using Google's search engine to access primary and secondary sources. We took steps to ensure a thorough search by training four research assistants to look through 10 pages of Google search returns, per search term, as well as any relevant sources cited therein. A source was considered relevant and included in our dataset if it contained narrative descriptions on the inner-workings of $\mathrm{VW}$ related to (1) the creation and refinement of the defeat device, (2) the implementation of the defeat device, and (3) the lengthy concealment of the defeat device. Sources included legal documents, investigative journalist reports, technical reports, employee interviews, and popular press articles.

\subsection{Timeline Construction}

In order to focus on the most salient actions of VW as they related to the creation, implementation, and cover up of the defeat device, we constructed a timeline of relevant events and links to sources. We identified key actors (e.g., VW managers and engineers) along with actions that pertained to the creation, implementation, and concealment of the defeat device. To ensure that the timeline was credible, research assistants were tasked with being devil's advocates and challenging both the emerging narrative and the credibility of sources of the narrative. Additionally, the authors critiqued the timeline and gathered more information as needed or directed the research assistants to do so.

\subsection{Data Analysis and Model Construction}

In order to build a causal model that explains how collaborative cheating emerges, we used grounded theory [22], which "begins with observations or data and seeks to induce empirical generalizations and concepts that reflect the contours or features of data" [13]. Further, drawing on Stake's guidance for case-study research [20], we bounded the case by focusing on generating concepts that explain how collaborative cheating emerged at $\mathrm{VW}$. We used our timeline as a guide for immersing ourselves in the data. While seeking to draw inferences, we also ensured that the supporting evidence was appropriate for a given event. For instance, one of our sources linked VW's behavior to policy issued by the EPA, but did not cite the EPA directly. So we identified the EPA document that issued this policy. Over time, we refined the data and our timeline to more closely align the two and, following Stake [20], clarified event descriptions to draw readers' attention to structural and procedural factors relating to the emergence of collaborative cheating at VW. In drawing inferences, we drew on the highest quality data in our dataset, which often meant drawing directly from legal documents. Articles from the popular press often corroborated the narrative we constructed, leant us possible interpretations of the facts, or served as a resource that helped us identify more credible facts (e.g., locating additional cases/legal proceedings, interviews, etc.). Once these events were constructed, they were clustered into themes, which informed the construction of a causal model explaining how collaborative cheating emerges (see Figure 1). Lastly, drawing on the open-systems approach [12], we built a causal model articulating the mechanisms that might explain how collaborative cheating emerged at VW and, correspondingly, how such phenomena might emerge in general.

\section{How Collaborative Cheating Unfolded at VW}

In crafting our timeline, it quickly became apparent that the behavior occurring at VW was not an isolated, one-time event. Rather, it was an ongoing effort reflecting many decision points faced by multiple VW employees from various departments and hierarchical levels who were making unique contributions over a substantial period of time. Consistent with our original intentions, we viewed the scandal as unfolding in three stages: (1) the creation of the defeat device [1999-2006], (2) the implementation and refinement of the defeat device [2007-2013], and (3) the concealment (and revelation) of the defeat device's existence [2014-present]. Table 1 lists those events most relevant to our analysis and links these events to emergent themes (our complete timeline, along with supporting data, is available for interested readers as supplemental online material. See https://osf.io/w8zyx/). We now summarize key events from these three periods.

\subsection{Creation of the Defeat Device}

In the 1990s, with the amendment of the Clean Air 
Act, the U.S. began a process of tightening emissions regulations, with the phase-in of new standards scheduled for 2004. Independently, in 1999, Audi engineers created software that could defeat European emissions testing. In 2006, following threats from VW AG's CEO (Executive $A$ ) - who had a reputation for firing engineers who did not meet his exacting standards - that employees would lose their jobs if VW could not sell diesel engines in the U.S, two department heads (Manager E, Head of VW's Engine Development After-Treatment Department and Manager D, Head of Diesel Engine Development for $V W$ ), knowing both that their engines exceeded emissions standards and how U.S. regulators would test VW vehicles, led the team that would create the defeat device. During this time, a VW employee acquired software from Company $A$ (an automotive engineering company specializing in software, electronics, and technology which VW owned $50 \%$ of) to create the EA189 engine that would be capable of defeating emissions testing.

Several themes emerge from this stage in the scandal. The creation of the defeat device software by Audi, a subsidiary of VW, suggests that the VW leadership developed a pervasive culture that encouraged the use of unethical means to attain market superiority. While the unethical leadership literature draws attention to how such leaders encourage subordinates to use any means to achieve market superiority [23], little field data exists examining how unethical leadership encourages individuals to collaboratively cheat, a point to which we now turn.

Moving closer to the creation of the defeat device, our data suggest that authority or bullying from leadership along with normative pressures can drive individuals within a group to decide to contribute to a collaborative cheating effort. While the influence of authority and conformity on individual decisions are fairly well-documented social psychological phenomena (e.g., administer lethal shocks, deliver incorrect answers in a group setting to avoid standing out, go along with a bad idea) [24-26], their role in causing collaborative cheating is not. Our data suggest that authority and normative pressures drove employees to collaborate out of short-term self-interest, generating solutions that were desirable from the perspective of their superiors'. Such findings are mirrored in the available literature [27]. However, this literature has not suggested a link between such organizational structures and collaborative cheating. The data we have covered suggest that unethical leaders can promote collaborative cheating by creating a climate of fear that facilitates employees' collective moral disengagement from ethical obligations [28]. In other words, leaders motivate employees to cheat, not for VW, but to spare themselves (at least, in the short-term). Scholars examining causes of unethical pro-organizational behavior $[4,5,6]$ might be best positioned to study this phenomena (see 5 for an exemplar). Additionally, while a wealth of literature exists on unethical or destructive leadership [23,29], we identified only one study linking such leadership to creative problem solving and it demonstrated that abusive leadership, which is apparent in our data, cascades down organizational hierarchies (consistent with our perspective), and ultimately undermines organizational creativity [30]. Our data suggest that such leaders might also cultivate a collective bottom-line mentality among employees, resulting in collaborative ventures that produce unethical solutions.

\subsection{Implementation and Refinement of the Defeat Device}

In 2007, after Manager $E$ and Manager $D$ authorized the use of the defeat device, Manager $E$ and $V W$ employees presented their newly developed engine to the Environmental Protection Agency (EPA) and California Air Resources Board (CARB) in order to obtain approval for marketing diesel vehicles to the U.S. In this meeting, Manager $E$ and $V W$ employees knowingly omitted and concealed information regarding the defeat device's existence. Around the same time, Company $A$ warns $V W$ executive leadership about the illegalities of using defeat devices, though later works with VW to implement the software. Later in 2007, Manager E, Manager D, and $V W$ employees meet to discuss which team is ultimately responsible for ensuring that the EA189 engine satisfies emissions standards. The meeting ends with Manager $E$ arguing to continue developing the EA189 engine with the defeat device and Manager $D$ formally authorizing the deployment of the EA189 engine. After presenting the EA189 engine to $V W$ executives in a presentation that supposedly omits mention of the defeat device, Managers $E$ and $D$ agree to remain silent about the defeat device. Engineer $B$, who worked on the team that developed the defeat device, later moves to the U.S. to oversee testing of the new diesel vehicles. In 2011, a supervisor (Supervisor A, VW AG's Quality Management and Product Safety supervisor) identifies the defeat device and raises concerns to Manager $B$ (a department head) regarding the illegality/risk/consequences of using the device. Supervisor $A$ later becomes complicit in the 
concealment of the device. After receiving a letter from VW engineers suggesting that the defeat device stresses the exhaust system resulting in hardware failures, Supervisor A and Manager B, the head of engine development for the VW Passenger Cars brand, instruct the engineers to destroy said letter. Supervisor $A$, Manager $B$, and $V W$ engineers then activated a "steering wheel angle recognition" feature to address the hardware failure, which allowed the vehicle to detect when it was undergoing testing or being driven on the road (when it no longer needed to operate in a low-emissions mode) and virtually ensured that it would go undetected using conventional laboratory methods (i.e., via dynamometer). $V W$ engineers expressed concerns to management, particularly Manager B, about this software update. Nevertheless, Manager $B$ authorized the installation of the function and instructed $V W$ employees to submit applications for the EPA to certify their vehicles.

The evidence emerging from this stage helps explain how the number of employees contributing to a collaborative cheating effort increased over time what we call an enveloping effect. The defeat device stressed equipped vehicles, indirectly revealing its existence to naive audience members. It is worth pointing out that the team that designed the defeat device at VW was aware that hardware failures would occur. Perhaps the team hoped to find a better solution in time (which we admit is mere speculation at this point in time). Nevertheless, when hardware failures occurred, those responsible for the defeat device had to address this issue. At this point in the timeline, many individuals had already contributed to the effort (or were still doing so), so for individuals uncovering the existence of the defeat device, a cost-benefit judgment had to be made in regard to contributing to the device's implementation. The costs of failing to contribute (e.g., losing one's job, threatening one's peers' or superiors' livelihoods) were probably seen as excessively high. Subsequently, future detections brought with them an increasingly larger number of contributors, further increasing pressures to contribute to the defeat device's ongoing use by raising costs of nonconformance. This strengthened the climate of fear and norms for conformity, virtually ensuring that the effort would envelop more people. Such an enveloping effect would be well-explained by threshold models of collective behavior [31], which note that individuals will join collective efforts when perceived benefits outweigh perceived costs of not doing so (e.g., loss of one's job, displeasing one's supervisors). Such models also explain the influence of authority, power, and conformity that were discussed previously and the clear escalation of unethical commitments that we see at this point in the case [32,33]. They may also explain how cooperation can promote corruption when partners' self-interests are aligned - more people simply get involved, raising the perceived costs of nonconformance [34].

\subsection{Ongoing Concealment (and Revelation) of the Defeat Device}

The last stage of our narrative begins in 2014, when a study sponsored by the International Council on Clean Transportation (ICCT) revealed that VW diesel-burning vehicles emitted NOx at levels between 5 and 35 times the allowed limit. VW headquarters in Wolfsburg elected to continue refining the device while also appearing to cooperate with U.S. regulators (i.e., the EPA and CARB). VW issued a recall of 500,000 vehicles and promised to address the emissions anomaly. However, part of the recall consisted of a software update to refine the defeat device's ability to evade detection.

Going into 2015, executive leadership engaged in the following activities that enveloped more employees in the overall collaborative cheating effort: (1) They restricted communications within VW to prevent employees from revealing any awareness of a defeat device, (2) they provided false explanations to regulators for emissions non-compliance (e.g., "irregularities" and "abnormalities," (3) they formed ad-hoc task forces to fabricate false reasons for the emissions anomaly, and (4) they continued to apply for approvals from the EPA to sell their emissions non-compliant vehicles in the U.S. Eventually, an unnamed employee (a VW executive in the U.S. responsible for emissions compliance) neglected executive instruction to keep the device concealed and explained the device's existence to CARB, forcing VW executives to formally reveal the software and bringing the device's concealment to an abrupt end.

This final stage in the scandal reveals a clear escalation of unethical organizational commitments involving leadership that envelops all relevant VW employees, and once revealed, damages VW's reputation. This outcome would be foreseen by Cialdini [35], who proposed that each act of organizational dishonesty raises the probability that dishonesty will be found out, causing reputational damages. He also argued that organizational deceptions can affect employee turnover by encouraging employees who are uncomfortable with engaging in unethical behavior to leave while those who are 
comfortable with dishonesty remain, further increasing the probability of dishonest acts. Additionally, Cialdini argued that organizational leaders' paranoia will increase, leading them to monitor employee behavior and create a climate of mistrust that harms firm performance. After considering our data from all three stages, it is clear that leaders' monitoring behavior increased, particularly once there was evidence indicating the existence of a defeat device. Though supporting one of Cialdini's claims, our data go one step further and suggest what we are tentatively calling a corrupting effect - VW leadership appeared to have turned a large number of employees who were initially uncomfortable contributing to the defeat device's ongoing use into contributors to the collaborative cheating effort. We suspect that all of the social factors we have discussed thus far (e.g., abuse, conformity, number of individuals contributing to the collaborative cheating effort) helped employees to perceive high costs of not contributing to the collective cheating effort, making it easier to morally disengage from their ethical responsibilities. If this corrupting effect is real, then much research is needed to understand its boundary conditions and to prevent these effects from emerging in organizational life.

\section{How Collaborative Cheating Emerges in Organizations}

Our overall causal model (see Figure 1), which is both grounded in our data and informed by the literature, suggests that collaborative cheating emerges as a consequence of organizational leadership that, in response to constraints on organizational performance, issues credible threats to subordinates. An ethically permissive organizational culture (which is not independent from leadership) and the number of collaborators, which increase over time, play similar causal roles: they cultivate a climate of fear where employees' job security is threatened [36] and a collective bottom line mentality where employees' self-interests become aligned [34], motivating them to pursue strategies that would secure their short-term self-interests. If a culture is ethically permissive, then unethical solutions may already exist, helping the collaborative cheating effort to emerge. As the collaborative cheating effort unfolds over time, more actors become aware and, upon appraising the costliness of failing to contribute, join the collective cheating effort. They then morally disengage (i.e., rationalize) contributing to the effort. As the number of individual contributors increases, (1) the variety of expertise within the collective effort increases, raising the group's ability to refine and sustain further collaborative cheating and (2) more people become at risk of reaching their threshold for joining the collective effort. Those who contribute become invested in sustaining the collaborative cheating effort. Hence, rather than leaving because they have been encouraged to violate their values, they remain. With a greater number of individuals contributing to the collective cheating effort(s), the organization's use of deceptive tactics increase (e.g., fraudulent products or services), raising the risk that falsehoods will be found out, resulting in organizational decline.

\section{Discussion}

Our case study suggests that collaborative cheating is more likely to occur when organizational cultures and the leaders that establish them create a social context (e.g., climate of fear, collective bottom line mentality) that motivates employees to use any means necessary to achieve market superiority. We find that such leadership functions much like a tumor [35], causing organizational behavior that leads employees who seemingly have a moral compass (e.g., expressing displeasure with contributing to a collective cheating effort) to collaboratively cheat. Our study contributes to research on collective forms of corrupt behavior by showing how institutional structures (e.g., leadership) and social factors (e.g., culture, climate, norms) might foster collaborative cheating, addressing a call for research into collective forms of unethical behavior [10].

\subsection{Theoretical and Practical Implications}

The behavioral ethics literature has been long informed by an ethical calculus perspective [9], which suggests that cost-benefit estimates inform the decision to cheat $[7-9,11]$. In this framework, individuals are likely to cheat if the perceived benefits of doing so outweigh the perceived costs. Another framework, grounded in social norms, suggests that the social context informs such decision making [8,37-40]. By explaining collaborative cheating behavior as an outcome of a cost benefit analysis that is informed by normative considerations [31], our study highlights a deep relationship between these two perspectives. Practically speaking, our findings suggest that remedies for collaborative cheating will be difficult to craft. Once organizational leaders commit to an unethical action, escalation of further commitments 
will likely follow, generating collective moral disengagement, and enveloping others, leading to ever riskier behavior (e.g., targeting regulations and regulators themselves). Organizational members may even grow persistently resistant to accusations of unethical behavior. Indeed, at least one VW executive denied in an interview that the VW scandal concerned a breach of ethics. While an obvious solution seems to be a change of leadership (e.g., top management team) and governance (e.g., board of directors), bringing this about is not so straightforward.

\subsection{Strengths, Limitations, and Directions for Future Research}

Though we aimed to systematically collect and analyze data to allow replicability, our study has many limitations that directly impinge upon our data quality and methodology. To address quality issues, we relied primarily on legal documents to craft our narrative. However, given the nature of our case (i.e., organizational deception) there is no guarantee that VW employees providing data on VW's inner-workings did not commit perjury. As there are ongoing investigations, more facts will emerge, which may call into question our findings and causal model. Therefore, our model should be viewed as preliminary and will be updated as more facts emerge. In regard to the generalizability of our process model, as our study consisted of a single case inside a German automaker, cultural-, industry-, and company-specific factors (to name a few) likely constrain the generalizability of our model. It is likely the case that our model contains particularisms that constrain generalizability. However, given that other organizations within the automotive industry are facing emissions cheating scandals (e.g., Fiat-Chrysler, General Motors), future research extending our methodology to these organizations would test the credibility of our model.

\section{References}

[1] A. C. Edmondson and K.S. Roloff, "Overcoming barriers to collaboration: Psychological safety and learning in diverse team," in E. Salas, G. F. Goodwin, \& C.S. Burke's (Eds) Team Effectiveness in Complex Organizations: Cross-Disciplinary Perspectives and Approaches, Routledge, New York, 2009, pp. 183-208.

[2] A.R. Jassawalla and H.C. Sashittal, "An examination of collaboration in high-technology new product development processes," Journal of Product Innovation Management, vol. 15, pp. 237-254, 1998.
[3] M.J. Pearsall and A.P.J. Ellis, "Thick as thieves: The effects of ethical orientation and psychological safety on unethical team behavior," Journal of Applied Psychology, vol. 96, pp. 401-411, 2011.

[4] E.E. Umphress, J.B. Bingham, and M.S. Mitchell, "Unethical behavior in the name of the company: The moderating effect of organizational identification and positive reciprocity beliefs on unethical pro-organizational behavior," Journal of Applied Psychology, vol. 95, pp. 769-780, 2010.

[5] C.M. Castille, J.E. Buckner, and C.Thoroughgood, "Prosocial citizens without a moral compass? Examining the relationship between Machiavellianism and unethical pro-organizational behavior," Journal of Business Ethics, 2016.

[6] M. Chen, C.C. Chen, and O.J. Sheldon, "Relaxing moral reasoning to win: How organizational identification relates to unethical pro-organizational behavior," Journal of Applied Psychology, 2016.

[7] M.G. Allingham and A. Sandmo, "Income tax evasion: A theoretical analysis," Journal of Public Economics, vol. 1, pp. 323-338, 1972.

[8] J. Hill and R.A. Kochendorfer, "Knowledge of peer success and risk of detection as determinants of cheating," Developmental Psychology, vol. 1, pp. 231-238, 1969.

[9] J.J. Kish-Gephart, D.A. Harrison, and L.K. Treviño, "Bad apples, bad cases, and bad barrels: Meta-analytic evidence about sources of unethical decisions at work," Journal of Applied Psychology, vol. 95, pp. 1-31, 2010.

[10] L.K. Treviño, G.R. Weaver, and S.J. Reynolds, "Behavioral ethics in organizations: A review," Journal of Management, vol. 32, pp. 951-990, 2006.

[11] L.K. Treviño and G.R. Weaver, "Punishment in organizations; descriptive and normative perspectives", in M. Schminke (Ed.) Managerial ethics: Moral management of people and processes, Lawrence Erlbaum, Mahwah, pp. 99-114. 1998.

[12] T.G. Cummings and C.G. Worley, Organizational Development and Change, 10th ed.

[13] K.M. Eisenhardt and M.E. Graebner, "Theory building from cases: Opportunities and challenges," Academy of Management Journal, vol. 50, pp. 25-32, 2007.

[14] R.P. Gephart, "Doing research with words: Qualitative methodologies and Industrial/Organizational Psychology," in J.M. Cortina and R.S. Landis (Ed.) Modern Research Methods for the Study of Behavior in Organizations, Routledge, New York, pp. 265-318, 2013. 
[15] J.W. Creswell, Qualitative inquiry and research design: Choosing among five approaches, Thousand Oaks, Sage, 2013.

[16] J.M. Cortina, H. Aguinis, and R.P. Deshon, "Twilight of dawn or of evening? A century of research methods in the Journal of applied psychology," Journal of Applied Psychology, 2017.

[17] G.J. Thompson, D.K. Carder, M.C. Besch, A. Thiruvengadam, and H.K. Kappanna, "In-use emissions testing of light-duty diesel vehicles in the United States", Research report.

[18] S.R.H. Barrett, R.L. Speth, S.D. Eastham, I.C. Dedoussi, A. Ashok, R. Malina, and D.W. Keith, "Impact of the Volkswagen emissions control defeat device on US public health," Environmental Research Letters, vol. 10, 2015.

[19] R.K. Yin, Applications of Case Study Research, Thousand Oaks, Sage, 2003.

[20] R.E. Stake, "Qualitative case studies," In N. K. Denzin and Y. S. Lincoln (eds) Strategies of Qualitative Inquiry. Sage Publications, Los Angeles, 2008.

[21] C. Moore, J.R. Detert, L.K. Treviño, V.L. Baker, \& D.M. Mayer, "Why employees do bad things: Moral disengagement and unethical organizational behavior," Personnel Psychology, vol. 65, pp. 1-48, 2012.

[22] B. Glaser and A. Strauss, "The discovery of grounded theory," Chicago, Aldine Press, 1967.

[23] S. T. Hunter, “(Un)Ethical leadership and identity: What did we learn and where do we go from here?" Journal of Business Ethics, vol. 107, pp. 79-87, 2012.

[24] S. Milgram, "Obedience to authority: An experimental view," New York: Harper \& Row, 1974.

[25] S. Asch, "Studies in independence and conformity," Psychological Monographs, vol. 70, no 416.

[26] I.L. Janis, Victims of groupthink: A psychological study of foreign-policy decisions and fiascoes. Boston: Houghton Mifflin, 1972.

[27] D. Kuyumcu and J.J. Dahling, "Constraints for some, opportunities for others? Interactive and indirect effects of Machiavellianism and organizational constraints on task performance ratings," Journal of Business and Psychology, vol. 20, pp. 301-310, 2014

[28] A. Bandura A, "Moral disengagement in the perpetuation of inhumanities," Personality and Social Psychology Review, vol. 3, pp. 193-209, 1999.
[29] D.V. Krasikova, S.G. Green, and J.M. LeBreton, "Destructive leadership: A theoretical review, integration, and future research agenda," Journal of Management, vol. 39, 1308-1338, 2013.

[30] D. Liu, H. Liao, and R. Loi, "The dark side of leadership: A three-level investigation of the cascading effect of abusive supervision on employee creativity," Academy of Management Journal, vol. 55, 1187-1212, 2012

[31] M. Granovetter, "Threshold models of collective behavior," American Journal of Sociology, vol. 83, pp. 1420-1443, 1979

[32] B.M. Staw, "The escalation of commitment to a course of action," Academy of Management Review, vol. 6, pp. 577-587, 1981.

[33] C.R. Greer, and G.K. Stephens, "Escalation of commitment: A comparison of differences between Mexican and US decision-makers," Journal of Management, vol. 27, pp. 51-78, 2001.

[34] O. Weisel and S. Shalvi, "The collaborative roots of corruption," Proceedings of the National Academy of Sciences of the United States of America, vol. 112, pp. 10651-10656, 2015.

[35] R.B. Cialdini, "Social influence and the triple tumor structure of organizational dishonesty," In D.M. Messick and A.E. Tenbrunsel's (Eds.) Behavior Research and Business Ethics, pp. 44-58, New York, Russell Sage Foundation, 1996.

[36] N.M. Ashkanasy and G.J. Nicholson, "Climate of fear in organizational settings: Construct definition, measurement, and a test of theory," Australian Journal of Psychology, vol. 55 , pp. 24-29, 2003.

[37] R.B. Cialdini and M.R. Trost, "Social influence: Social norm, conformity, and compliance," In D.T. Gilbert, S.T. Fiske, \& G. Lindzey (Eds.), Handbook of Social Psychology, vol. 2, pp. 151-192, New York: McGraw-Hill, 1998.

[38] A. Bandura, "Influence of models' reinforcement contingencies on the acquisition of imitative responses," Journal of Personality and Social Psychology, vol. 1, pp. 589-595, 1965.

[39] F. Gino, S. Ayal, and D. Ariely, "Contagion and differentiation in unethical behavior: The effect of one bad apple on the barrel," Psychological Science, vol. 20, pp. 393-398, 2009.

[40] H. Tajfel, J.C. Turner, "The social identity theory of intergroup behavior," In S. Worchel \& W.G. Austin (Eds.), Psychology of Intergroup Relations, pp. 7-24, Chicago: Nelson-Hall, 1986. 
Table 1. Table Containing Event Data and Emergent Themes

\begin{tabular}{lc}
\hline (\#Event Number) Summary of Event Data & Emergent Themes \\
\hline (\#4) EPA plans tighter emissions standards and sets a timeline for implementation. & Constraints on \\
(\#15) Diesel engines cannot both satisfy customer demands for power and comply with emissions & Performance/Behavior \\
standards. & \\
(\#48) $E P A$ and $C A R B$ refuse to approve $V W$ vehicles until $V W$ comes forward with explanations for & \\
emissions issues. &
\end{tabular}

(\#9) Audi engineers develop a prototypical device for defeating emissions testing, but it is not yet implemented.

(\#2) $V W$ was fined for employing defeat devices (in 1973).

(\#6 \& \#8) General Motors sues VW AG, Executive A, GM Executive A, and other GM employees over corporate espionage scandal. $V W$ forced to pay $\$ 100$ million dollars to $G M$ and to buy $\$ 1$ billion in components from one of GM's companies.

(\#12) "Perks and prostitutes" scandal comes to light. VW paid for "special bonuses" - including expenses for prostitutes - to the chairman of the VW workers council. Executive K, Chairman A, and Deputy $A$ all received prison sentences in the wake of the scandal.

(\#18) Executive A (CEO of VW AG), who was infamous for firing subordinates if they did not meet his exact standards, threatens engineers promising to fire them if they cannot sell diesel engines in the U.S.

Ethically Permissive

Organizational Culture

(\#17) Two heads of separate departments (Manager E \& Manager D), knowing how the vehicles will be tested and recognizing that vehicles cannot satisfy power, timing, and emissions constraints, direct employees to design, create, and implement an engine capable of cheating the emissions test. Engineer $B$ leads the team, which obtains software from Company $A$ that is turned into the defeat device. After being informed of the defeat device's use, they (Manager E \& Manager D) authorize its use.

(\#26) Engineer $B$ moves to U.S. to oversee development of vehicles fitted with defeat devices. (\#31) Supervisor $A$ and Manager $B$ instruct $V W$ engineers to destroy document explaining how defeat device causes hardware failures.

(\#34) Manager B authorizes improvements to the defeat device software.

(\#39) Manager C calls a VW employee "crazy" for sending out an email implying knowledge of the defeat device, its concealment, and implicating Manager $C$ and Manager B.

(\#51) Manager $C$ misleads $C A R B$ by suggesting that increased emissions were due to

"irregularities" and "abnormalities" rather than a defeat device.

(\#46 \& 47) Liaison A encourages $V W$ employees to think of false reasons why $V W$ vehicles emit excessive emissions. Reiterates in a later email that they need "good arguments" to counter questions from U.S. regulators.

(\#50) Executive $F$ provides false information pertaining to $V W A G$ 's total liabilities, profit, assets, and shareholder's equity.

(\#52) Manager B and other co-conspirators give a script to employees meeting with CARB. This script specifies further concealment of the defeat device and instructs employees to obtain approval from CARB to sell newer vehicles that contain defeat devices. (\#59) Executive $C$ denies that the scandal is an ethical problem.

(\#17) Company A shares prototypical defeat device software with the team responsible for creating what became known as VW's defeat device.

(\#19) Manager $E$ and employees present EA 189 engine to $E P A$ and $C A R B$ without disclosing defeat device's existence.

(\#22 \& 23) Manager D and Manager E present EA 189 engine to executives, omitting information about the defeat device.

(\#24) Manager D tells Manager E they will never present the information on the defeat device to anyone.

(\#37) Manager $C$ notifies Supervisor $A$ that ICCT members suspect cheating.

Abusive Supervision

(\#38) Manager B, Supervisor A, Manager C, Liaison A, and co-conspirators, responding to questions from $C A R B$ in coordination with the EPA, elect to not disclose the defeat device while appearing to cooperate with U.S. regulators.

(\#44) VW headquarters in Wolfsburg uses a recall to refine the defeat device.

Selective Information Sharing and also Complicit Deception by Means of Omission and Fabrication 
(\#51) Manager $C$ deceives $C A R B$ citing technical reasons such as "irregularities" and

"abnormalities".

(\#52) Manager B and other co-conspirators provide a script to employees who meet with CARB.

The script encourages further concealment of the defeat device, which will allow VW to obtain approval to sell newer vehicles that still contain the defeat device.

(\#20) Company $A$ warns $V W$ executives (including Executive $B$ ) that the defeat device is illegal. Later, works with $V W$ employees to create defeat device.

(\#28) Supervisor A explains illegality of defeat device to Manager B along with risks and consequences of using device.

(\#31) VW engineers give document to Supervisor A and Manager B explaining how the defeat device stresses the exhaust system, resulting in hardware failures.

(\#33) $V W$ engineers express ethical concern to management about refined defeat device, but sought approval from senior management (Manager B).

(\#53) VW employee neglects instructions from management, revealing both the existence of the defeat device and the conspiracy to cheat the emissions test to $C A R B$.

(\#21) Manager $E$ and Manager D disagree over the team responsible for ensuring emissions standards.

Expressions of Ethical

Concerns (and

Subsequent Relapse) -

Moral Disengagement

(\#21) Manager E argues that production of the EA189 engine should continue. Manager D authorizes Manager $E$ to implement defeat device.

(\#31) Supervisor $A$ and Manager $B$ instruct $V W$ engineers to destroy document explaining how defeat device causes hardware failures.

(\#32) VW engineers, responding to Supervisor A and Manager B's instructions to destroy document explaining how the defeat device causes hardware failures, modify the defeat device by activating a steering wheel angle recognition feature.

(\#34) Manager $B$ authorizes improvements to defeat device software.

(\#51) Manager $C$ misleads $C A R B$ by suggesting that increased emissions were due to

"irregularities" and "abnormalities" rather than a defeat device.

(\#46 \& 47) Liaison A encourages $V W$ employees to think of false reasons why $V W$ vehicles emit excessive emissions. Reiterates in a later email that they need "good arguments" to counter questions from U.S. regulators.

(\#50) Executive $F$ provides false information pertaining to $V W A G$ 's total liabilities, profit, assets, and shareholders' equity.

(\#52) Manager B and other co-conspirators provide employees meeting with CARB with a script that encourages further concealment of the defeat device and might attain approval to sell newer vehicles that still contain defeat devices.

Note: $\#$ - refers to the event number found in the supplemental timeline (https://osf.io/w8zyx/), which is placed here for the reader to reference if needed. Additionally, data sources are included for each event in the timeline.

\section{Figure 1. How Collaborative Cheating Emerges in Organizations}

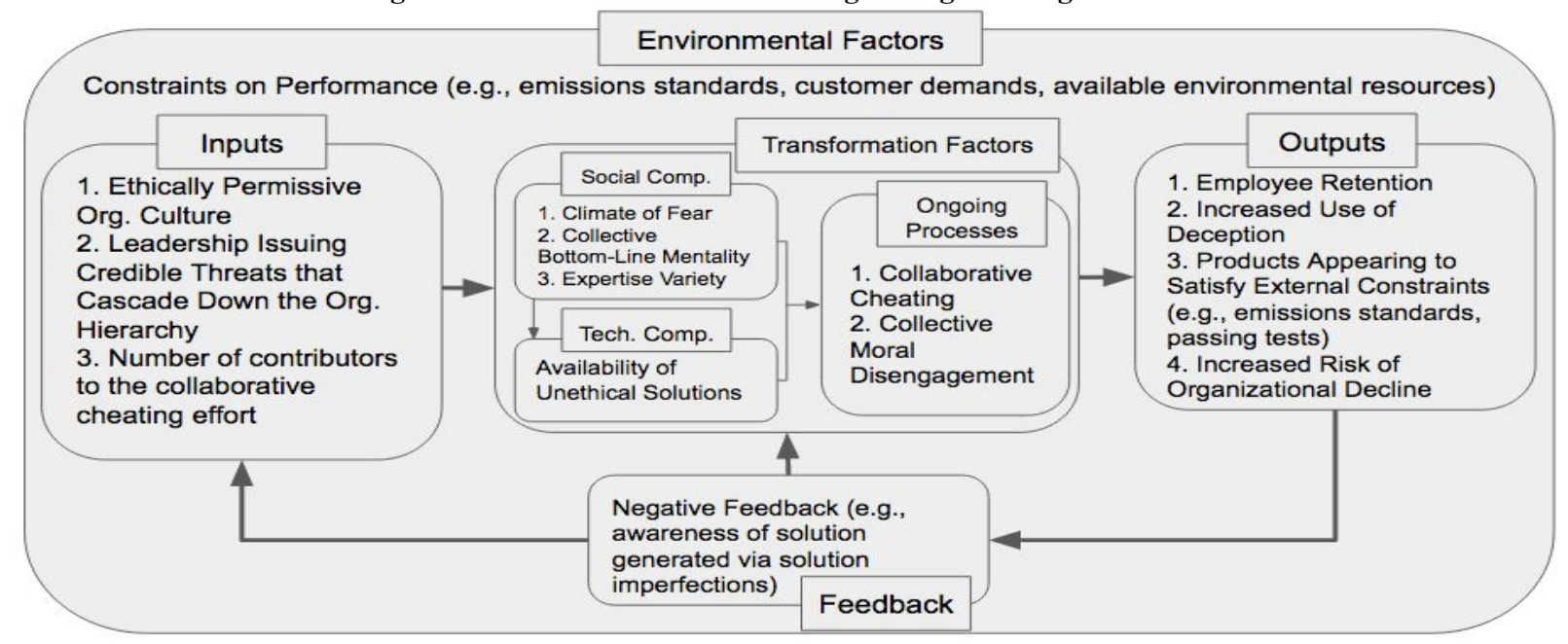

